IP Periodica Polytechnica Chemical Engineering

\author{
62(1), pp. 1-7, 2018 \\ https://doi.org/10.3311/PPch.10143 \\ Creative Commons Attribution (1)
}

RESEARCH ARTICLE

\section{Quantitative Evaluation of Drug Distribution in Tablets of Various Structures via Raman Mapping}

\author{
Attila Farkas $^{1 *}$, Brigitta Nagy ${ }^{1}$, György Marosi ${ }^{1}$
}

Received 11 October 2016; accepted 31 January 2017

\begin{abstract}
Hyperspectral imaging was applied to provide quantitative spatial information about pharmaceutical samples. The aim was to characterize the distribution of active pharmaceutical ingredient (API) in manufactured samples quantitatively. Two kind of API, imipramine and spironolactone were applied in three conventional and two continuous processing technologies. The homogeneity of distributions of 10\% API was determined through Raman maps applying macropixel analysis method. Non-overlapping macropixel analysis (Poole-index) and calculation of distributional homogeneity indices (DHIs) were compared as a measure of homogeneity. Non-overlapping macropixel approach proved to be more sensible than DHI evaluation. For enhancing efficacy of DHI we suggest a correction by weighting scores and considering relative standard deviations. This way the capability of DHI can be improved significantly. The very slight differences between the continuous methods (the homogeneity of which are much higher than that of conventional technologies) could be quantified.
\end{abstract}

\section{Keywords}

Raman mapping, pharmaceuticals, homogeneity, macropixel analysis

\section{Introduction}

Chemical imaging is progressively becoming one of the best available techniques to study samples in solid form in a non-destructive way [1]. The vibrational spectroscopy such as Raman or NIR is potentially useful to provide qualitative [2] and quantitative [3-6] information about the studied substances. In addition, the application of imaging procedure allows us to obtain a number of local, pixel-like data in lateral or even vertical directions. This approach has become favoured and increasingly preferred in the field of material characterization. The pharmaceutical industry brings the Raman imaging into focus due to its advantages in local determination of chemical and physical characteristics along the original and generic research and development. Numerous papers have reported that this technique is well suitable for analysing polymorphic forms [7], exploring relationship of the ingredients or determining the composition of counterfeits $[8,9]$. There is a need for extending this method to investigate the distributional homogeneity of blends. During manufacturing processes the proper homogeneity has a key role, which determines important properties of the end product. For instance, the inappropriate distribution leads to lower mechanical strength of tablets, diverse stabilities or unacceptable disintegration time. The inhomogeneity of the active pharmaceutical ingredient (API) can lead to inacceptable content uniformity.

The analysis of a Raman map results in huge amount of (semi)quantitative values ordered in a two-dimensional way presenting the distribution of a desired component $[1,10]$. The elements of the map create a histogram and the fitted probability distribution is characterized by standardized moments (mean, standard deviation, skewness, curtosis). Amigo [11] presented in a study the weakness of these statistics demonstrating how misleading can be their usage on a hyperspectral image and that none of them is sufficient by itself to describe the homogeneity properly. On these grounds it seems to be not advisable to make any conclusion regarding the sample homogeneity. A more rational way is to compare the maps of real samples to a perfectly homogeneous ideal sample. Common definition of a perfectly random mixture defines the probability

\footnotetext{
${ }^{1}$ Department of Organic Chemistry and Technology, Faculty of Chemical Technology and Biotechnology, Budapest University of Technology and Economics, H-1111 Budapest, Budafoki út 8., Hungary

*Corresponding author, e-mail: farkas.attila@mail.bme.hu
} 
for finding a particle of a constituent identical at all points in the mixture [12]. Unlike a liquid mixture, the pixels of a Raman image in solid sample differ from each other depending on the particle size of the component. It is feasible to construct an artificial map from the real distribution considering its randomly distributed pixels homogeneous. The most adjacent pixels can be clustered and assessed as a macropixel. This approach called macropixel analysis has been already used for characterizing tablets and capsules in NIR-CI [13].The term macropixel refers to a square cluster of pixels with an intensity value equal to the average or the standard deviation value of the included pixels [13]. The quantitative analyses of the macropixels enable to assess spatial characteristics of drug products and pharmaceutical blends. Thus the macropixel analysis provides a quantitative way to evaluate the distributional homogeneity.

Further objective measures have been developed in recent years [14-16]. Rosas et al. [14] considered Poole-index as the most adaptable index for the hyperspectral imaging. They binarized at first the studied map using arbitrary threshold and worked with non-overlapping macropixels. Sacre et al. [15] developed another criterion called Distributional Homogeneity Index (DHI). This method is based on the approach of the Continuous-Level Moving Block (CLMB) [13]. Unlike non-overlapping macropixels, this method initially searches all macropixels of size $2 \times 2$ pixels then evaluates them. In the next step the macropixel size is increased. The method proceeds until the total map size is reached. Linear relationship has been observed between DHI values and content uniformity values [15]. For certain samples these methods proved to be very suitable for the purposes, however for other samples such approaches are not discriminative enough. For instance, the homogeneity of samples can be set to rank order according to Poole-index using one macropixel size. However, it is not clear how to rank the samples if the Poole-indices show different order at other macropixel sizes. The DHI values can discriminate the homogeneities only if the pixel values form very similar Gaussian distributions (in the cases of different batches of a product) but it is not clear what to do if this criterion is not fulfilled? Furthermore, comparison of two methods has not been published yet. This paper aims to assess the homogeneity of samples of different drug distribution (due to different manufacturing methods) utilizing data collected from Raman maps.

\section{Materials and methods}

\subsection{Samples}

The samples used for obtaining the Raman mapping datasets were described in two published papers $[17,18]$. The model drugs (imipramine and spironolactone) were applied in pharmaceutics manufactured differently. Each solid sample contained $10 \%$ of active pharmaceutical ingredient (API). The samples containing imipramine hydrochloride (IMP, antidepressant classified as BCS I, provided by EGIS Pharmaceuticals
Plc., Budapest, Hungary) with a particle size of $100 \mu \mathrm{m}$ were prepared by three kind of manufacturing technologies: direct tabletting (D), fluid-bed granulation by water (F-W) and highshear granulation with water (HS-W) [17]. Further ingredients were microcrystalline cellulose (FMC BioPolymer, Princeton, USA), maize starch (Colorcon, West Point, USA), hydroxypropyl-methyl-cellulose (Shin-Etsu, Tokyo, Japan) binder and magnesium stearate (Faci Spa, Carasco, Italy) lubricant. The mean particle sizes were $100,65,60$ and $10 \mu \mathrm{m}$, respectively. The samples containing spironolactone (SPL, potassium sparing diuretic Sigma-Aldrich, Budapest, Hungary) of poor water solubility ( $28 \mu \mathrm{g} / \mathrm{mL}$, BCS II) were produced by electrospinning and melt-extrusion methods. Polyvinyl caprolactame-polyvinyl acetate-polyethylene glycol graft copolymer (Soluplus, molecular weight $=\sim 118,000$ Da, BASF, Ludwigshafen, Germany) was selected as matrix polymer and applied in the procedure described recently [18].

\subsection{Raman measurements}

Raman spectra were collected using a Horiba Jobin-Yvon LabRAM (Longjumeau, France) system coupled with an external diode laser source (785 nm, $80 \mathrm{~mW}$ ) and an Olympus BX-40 optical microscope. The tablets containing imipramine were mapped with objectives of $10 \times$ and $100 \times$ magnification applying $10 \mu \mathrm{m}$ and $2 \mu \mathrm{m}$ step sizes respectively. The acquisition time of a single spectrum was $30 \mathrm{~s}$ in each experiment and two spectra were averaged at a time. The measured area, when the $10 \times$ objective was used, varied from $28 \times 28$ to $33 \times 33$ pixels. Maps recorded with the $100 \times$ objective consisted of $60 \times 60$ pixels. In the case of materials containing spironolactone low-resolution maps were measured using $10 \times$ magnification. These electrospun and melt-extruded (SPL) samples were analysed with $50 \mu \mathrm{m}$ step size. The measurement per sample was performed from $24 \times 24$ points and three spectra of $30 \mathrm{~s}$ were averaged per point. In each case the component concentrations were estimated with the classical least squares (CLSs) method [19] using the reference spectra of the pure components collected on the same device under the same conditions. Visualized score maps were created with LabSpec 5.41 (Horiba Jobin-Yvon). These scores were applied for macropixel analysis.

\subsection{Macropixel analysis}

The pixels can be assessed by macropixel analysis on a two-dimensional image. The analysis can be performed using Discrete-Level Tiling (DLT) method with non-overlapping tiles or Continuous-Level Moving Block (CLMB) method with overlapping macropixels. Along the procedures the macropixel size has to be increased step-by-step. The macropixel sizes are strictly limited in DLT defining divisors of the total map size. In case of CLMB the macropixel size can be increased by 1 pixel on each step until the whole distribution map size is reached. In both cases a randomized distribution map, created 


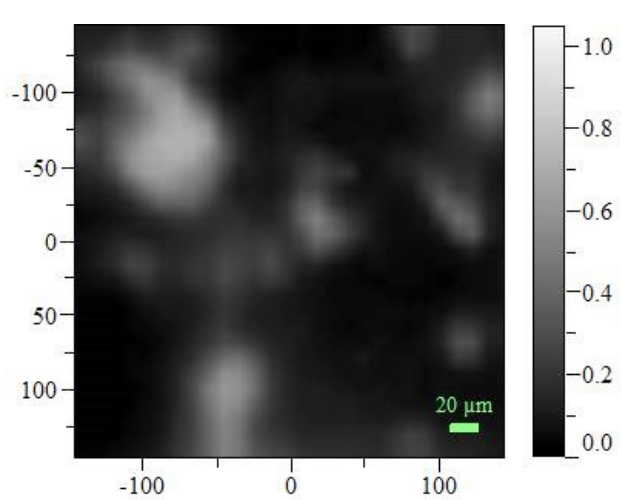

a)

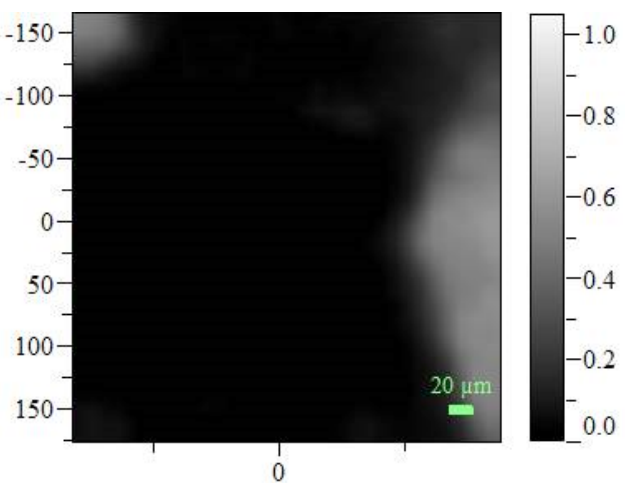

c)

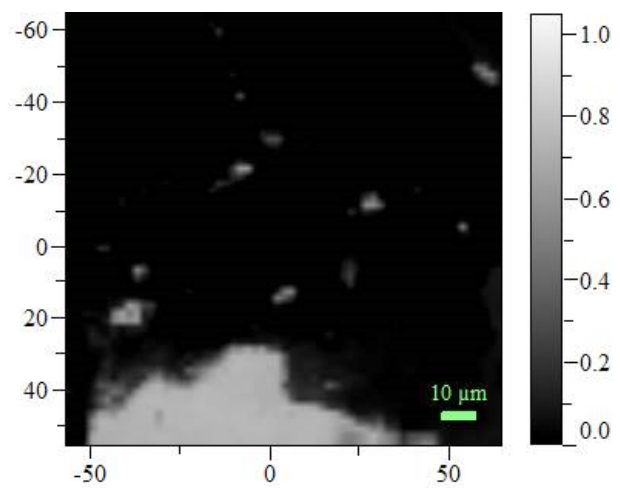

e)

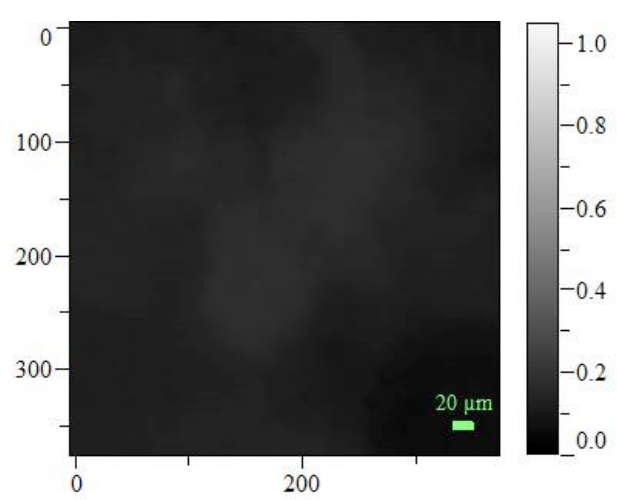

b)

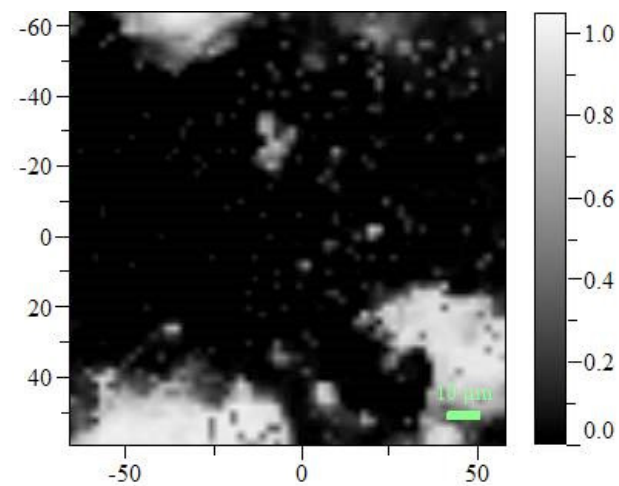

d)

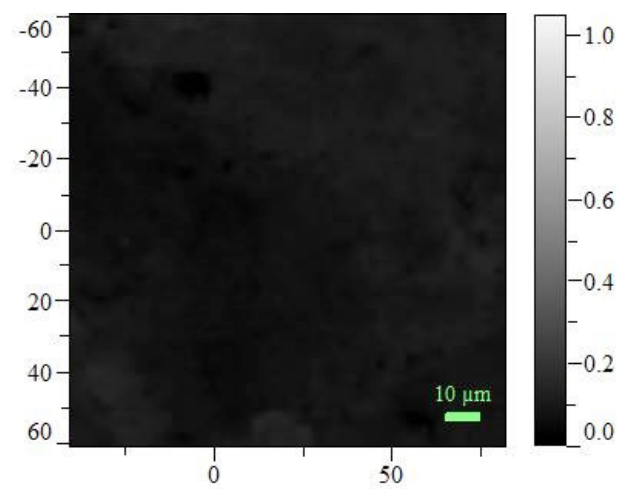

f)

Fig. 1 Spatial distribution of IMP in tablets manufactured by a) direct tabletting (D) b) high-shear granulation with water (HS-W), and c) fluid-bed granulation with water (F-W) as determined by low-resolution (10× objective) maps where the black area indicates the lack of API and the white area shows its high concentration level. Similar but high-resolution (100× objective) maps are shown in d) D, e) HS-W and f) F-W images

by random mixing of the original distributional map (in order to model the perfect homogeneous distribution), is needed to assess simultaneously. The original images can be compared to the randomized map.

\section{Results and discussions}

The distributions of drug in IMP-containing samples are shown on the Fig. 1.

Significant differences can be encountered, already after visual inspection, in the Raman maps of the tablets manufactured different ways. The maps prepared by $10 \times$ magnification (Fig 1.a-c) show similar distribution as the high-resolution maps (Fig 1.d-f). On the maps of D tablet (prepared by direct tableting) discrete particles can be seen. In the F-W tablet (granulated in fluid bed before tableting) larger aggregated particles are at the edge of the maps and API-free (black) areas can be found too.

In contrast, the Raman maps taken of the HS-W sample (granulated in high shear mixer) indicates higher homogeneity, although there was not enough time to dissolve IMP entirely in the granulating liquid (contour of the particles remained visible). Fig. 2 depicts the SPL distributions in extruded (Fig. 2.a) and electrospun (Fig. 2.b) samples.

These images show much better distribution comparing to the IMP samples. All these subjective impressions should be demonstrated with objective calculated measures. For this purpose, two evaluation methods were used for assessing sample homogeneities. 


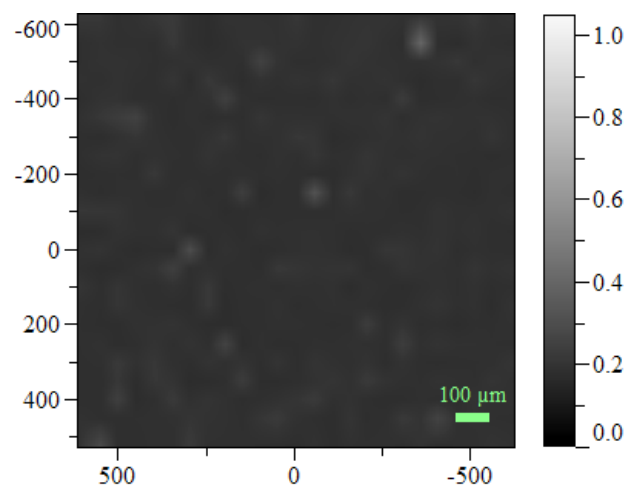

a)

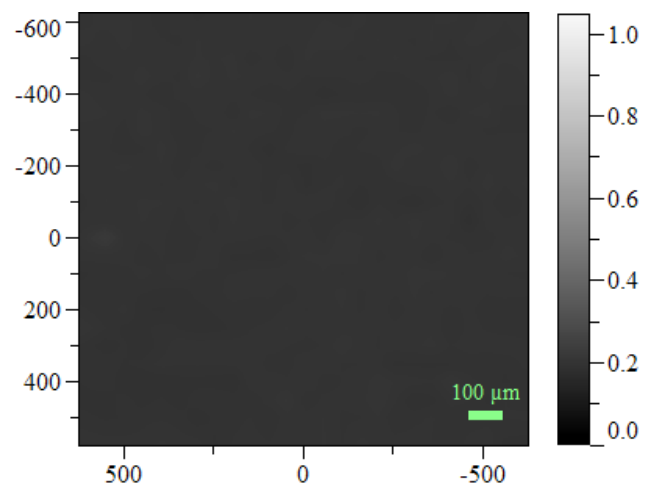

b)

Fig. 2 Spatial distribution of SPL in the a) extruded and b) electrospun samples

\subsection{Homogeneity evaluation based on the Poolelndex}

At first non-overlapping macropixels were generated from the CLS-score maps. The low-resolution IMP maps were prepared with various map size (as written in Section 2.2). In the case of $\mathrm{D}$ tablets the increasing macropixel size of 2, 4, 7 and 14 pixels (at the edge of macropixels) was set for a map of $28 \times 28$ pixels (shown in Fig. 1). Maps of $32 \times 32$ pixels relate to HS-W and F-W tablets (Fig. 1). The divisors of such map size were 2, 4, 8 and 16 pixels. In the case of SPL maps (Fig. 2) macropixels with divisors of 2, 3, 4, 6, 8 and 12 pixels were used on the map size of $24 \times 24$ pixels. Such approach has been applied for the high-resolution maps (Fig. $1 \mathrm{~d}$, e and f) as well, in which cases the $60 \times 60$ pixels were retained. The same non-overlapping macropixels have been also created on the randomized maps. In the next step the global mean standard deviation (GMSD) of the original and randomized image for macropixels was calculated [20] according to Eq. (1)-(3).

$$
\begin{gathered}
S D_{j}^{C}=\sqrt{\frac{\sum_{i=1}^{n}\left(c_{i j}-\bar{c}_{j}\right)^{2}}{n-1}} \\
G M S D=\frac{\sum_{j=1}^{m} S D_{j}^{C}}{m} \\
H \%_{\text {Poole }}=\frac{G M S D_{S M}}{G M S D_{R M}} 100,
\end{gathered}
$$

where is the standard deviation of the concentration in $j$ th macropixel; is the $i$ th element of the $j$ th macropixel; $n$ is macropixel size; $m$ is the number of macropixels on the sample map (SM) and the randomized map (RM).

The GMSD value for real map and the GMSD for the randomized map was divided and then multiplied by 100 to obtain the percentage homogeneity $\left(\mathrm{H}_{\mathrm{Poole}_{\mathrm{P}}}\right)$ in the Poole index according to Eq. (3). The Figs. 3 and 4 demonstrate the calculated $\mathrm{H} \%_{\text {Poole }}$ changings on the low-resolution and the high-resolution maps of IMP, respectively.

In the case of Raman maps with $10 \times$ magnification a relative macropixel area was needed to determine relating the

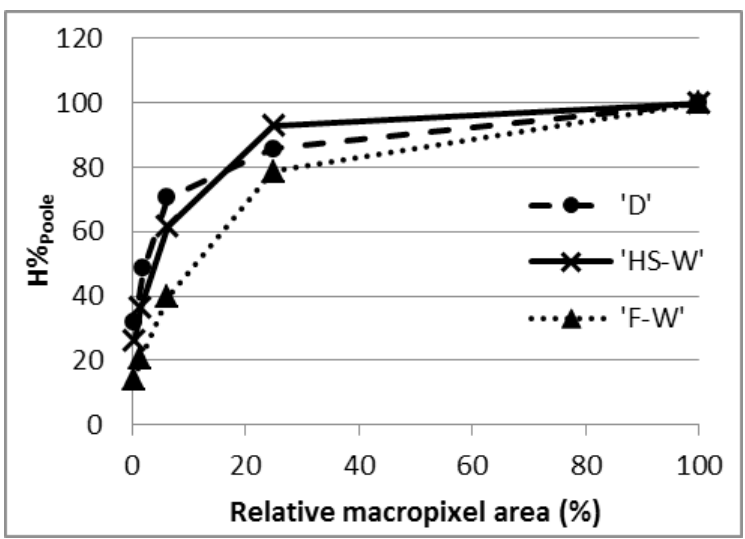

Fig. 3 Percentage homogeneity of Poole-index on the low-resolution maps (10× magnification) of different manufactured tablets:

D -direct compression, HS-W -high-shear granulation by water and F-W -fluid-bed granulation by water

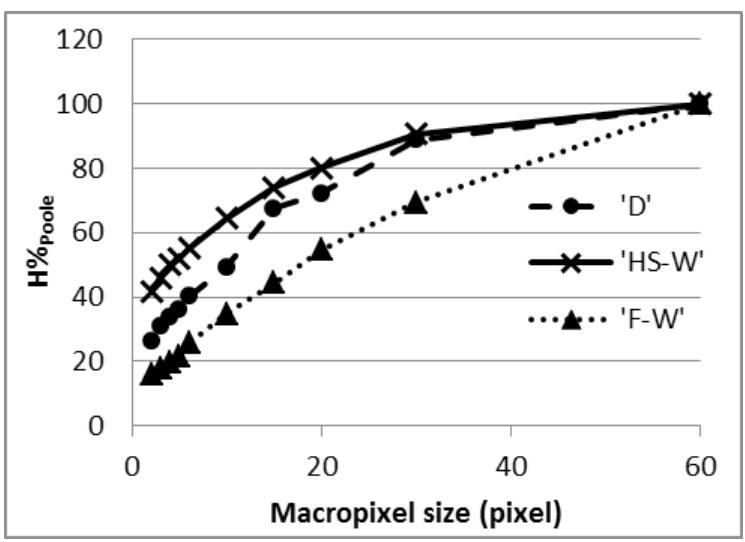

Fig. 4 Percentage homogeneity of Poole-index on the high-resolution maps (100× magnification) of different manufactured tablets: D -direct compression, HS-W -high-shear granulation by water and F-W -fluid-bed granulation by water

current macropixel size to the selected total map size due to its variation from sample to sample. This diagram indicates the markedly worse homogeneity of F-W comparing to the other samples. However, no remarkable difference could be observed between the D and HS-W. In contrast to these low-resolution maps, the distribution maps prepared by $100 \times$ magnification 
and lower step size has revealed much more relevant differences between the tablets. The homogeneity curve indicates that HS-W sample is superior to D sample at each macropixel size. The F-W has still lower $\mathrm{H} \%_{\text {Poole }}$ values than the other two cases confirming less homogeneity of API within that tablet.

The similarly calculated $\mathrm{H} \%_{\text {Poole }}$ indices for SPL samples are collected in Table 1. In samples manufactured by continuous technologies the homogeneity proved to be spectacularly enhanced. The indices at each macropixel size exceeded $90 \%$ in both technologies, which is considered homogeneous at the applied magnification. This $90 \%$ is achieved in both cases already at macropixel size 2 pixels, which means that any kind of inhomogeneity is lower than 1 pixel.

Table 1 Percentage homogeneity of Poole-index in SPL samples prepared by extruded and elctrospun technologies depending on the applied macropixel size

\begin{tabular}{lll}
\hline \multirow{2}{*}{ Macropixel size } & \multicolumn{2}{c}{$\mathrm{H}_{\text {Poole }_{\text {f }}}$ for SPL } \\
\cline { 2 - 3 } & Extruded & Electrospun \\
\hline 2 & 100.1 & 91.4 \\
3 & 98.7 & 94.1 \\
4 & 98.0 & 95.6 \\
6 & 98.7 & 96.8 \\
8 & 98.6 & 98.6 \\
12 & 99.2 & 99.6 \\
24 & 100 & 100 \\
\hline
\end{tabular}

\subsection{Homogeneity evaluation based on the Distributional Homogeneity Index}

DHIs were calculated for the same maps according to Eq. (4)-(6)

$$
\begin{gathered}
S D_{m}^{M}=\sqrt{\frac{\sum_{i=1}^{k}\left(\bar{c}_{i m}-\overline{\bar{c}}_{m}\right)^{2}}{k-1}} \\
A U C=\sum_{m=2}^{s} S D_{m}^{M} \\
D H I=\frac{A U C_{S M}}{A U C_{R M}},
\end{gathered}
$$

where $S D_{j}^{M}$ is the standard deviation of the mean concentration while the macropixel size is $m ; s$ is the map size; $m$ is macropixel size; $k$ is the number of macropixels $\left(k=(s-m+1)^{2}\right)$; $\bar{c}_{i m}$ is the mean value in $i$ th macropixel with $m$ macropixel size; $\overline{\bar{c}}_{m}$ is the mean value of $\bar{c}_{i m}$; AUC is the sum of standard deviations with different macropixel sizes (area under the curve) on the sample map (SM) and the randomized map (RM).

Selecting proper map size is unnecessary for these calculations in accordance with the approach of CLMB. However, due to better comparability the same map sizes were kept that have been used for GMSD calculations. DHI as a characteristic measure for the homogeneity has been calculated for the examined samples. The lower values in Table 2 represent higher homogeneity. The F-W sample shows the largest DHI values, which indicates poor homogeneity (in accordance with $\mathrm{H} \%_{\text {Poole }}$ calculations). However, in case of high-resolution maps the order of homogeneity differs from that of given by the Poole-index.

Table 2 Distributional homogeneity indices (DHI) in Raman distribution maps of the different manufactured samples

\begin{tabular}{lllll}
\hline \multirow{2}{*}{ API } & $\begin{array}{l}\text { Manufacturing } \\
\text { technologies }\end{array}$ & Objective & DHI & $\begin{array}{l}\text { Std. } \\
\text { dev. }\end{array}$ \\
\hline & direct compression (D) & $10 \times$ & 3.8 & 0.4 \\
& fluid-bed granulation & $100 \times$ & 5.4 & 0.4 \\
Imipramine & with water (F-W) & $100 \times$ & 6.9 & 0.4 \\
(IMP) & high-shear granulation & $10 \times$ & 4.1 & 0.3 \\
& with water (HS-W) & $100 \times$ & 7.1 & 0.5 \\
\hline Spironolactone & melt extrusion & $10 \times$ & 1.1 & 0.1 \\
(SPL) & electrospinning & $10 \times$ & 1.3 & 0.2 \\
\hline
\end{tabular}

Considerably lower DHIs can be seen on the melt-extruded and electrospun samples, thus DHI (similarly to Poole-index) is suitable for discriminating the advanced continuous methods from the other conventional manufacturing processes. However, CLMB calculations include a bias: Along this process the pixels are used in different number of macropixels. For instance, the frequency of selection per pixel is shown at a macropixel size of 4 in Fig. 5. The pixels in the corner are selected one time while moving towards to inside of the map the frequency of selection of the pixels is increased. The highest bias occurs if the macropixel size is the half of the map size. The authors therefore recommend that weighted means (wDHI) should be applied in DHI calculation to avoid bias. The weightings are the reciprocals of corresponding frequencies.

Furthermore, the characteristics of distributions should be considered as well when DHI values are intended to use for comparing homogeneities. The DHI is a very suitable measure when samples come from very similar distribution as it has been used for controlling content uniformities of certain batches in an earlier paper [15]. Nevertheless the DHI is a relative value due to the calculation method in which the real distribution is compared with an artificial randomized image. Therefore, it is unreasonable to expect that the similar DHI values indicate similar homogeneities while different distributions are examined. The method is not optimal to handle samples with strongly different distributions. The standard deviation of the original data, which includes substantial information, will be lost when real maps are compared to randomized images. Consequently, it is desirable to be calculated a corrected measure by multiplying the relative standard deviation (RSD) in order to avoid information loss and to facilitate the comparison of any kind of samples, such as materials manufactured differently. 


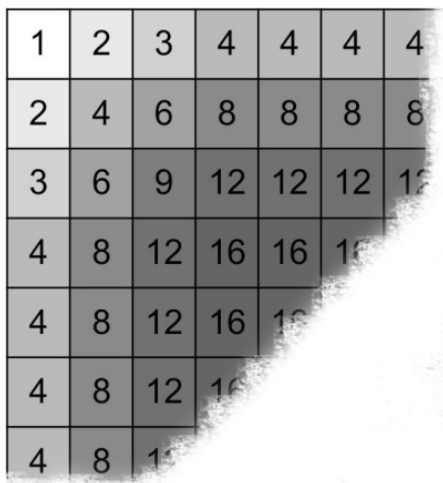

Fig. $5 \mathrm{~A}$ part of the selection frequencies of pixels into $4 \times 4$ macropixels on a distribution map using CLMB method

Accordingly, the DHI values have been reconsidered. First, the aforementioned weighting was applied for calculations of mean values in Eq. (4) then the RSD of maps were taken into account resulting in a corrected, cDHI, value. Table 3 summed up the results in this manner. The weighted DHIs (wDHI) increased significantly (comparing to the DHIs in Table 2) in those cases where particles or larger inhomogeneities are located at the edges of maps (Fig 1. c, d, f). The DHI of low-resolution HS-W maps decreased significantly to 6.5 and became similar like the $\mathrm{D}$ (6.3) discriminating it from the F-W (8.0). A perfect match could be achieved between the cDHIs and the visual inspections. The DHIs of F-W maps were the highest values of all cDHIs (5.3 and 12.2 in low-resolution and high-resolution maps, respectively). The $\mathrm{D}$ maps presented moderately high cDHIs of 2.1 and 7.9. In contrast, values below 1 could be achieved by HS-W technology. The homogeneity achieved through melt-extrusion and electrospinning exceeded the homogeneities provided by the conventional manufacturing technologies. The cDHIs were approximately 0.1 on the maps of melt extruded and electrospun samples. In addition the electrospun SPL sample is more homogeneous than melt-extruded one based on corrected DHIs.

Table 3 Weighted and corrected distributional homogeneity indices (DHI) and relative standard deviation (RSD) in Raman distribution maps of the different manufactured samples

\begin{tabular}{llllll}
\hline API & Manufacturing technologies & Objective & wDHI & RSD & cDHI \\
\hline \multirow{2}{*}{ direct compression (D) } & $10 \times$ & 3.3 & 0.63 & 2.1 \\
& & $100 \times$ & 6.3 & 1.25 & 7.9 \\
\multirow{2}{*}{ IMP } & $\begin{array}{l}\text { fluid-bed granulation } \\
\text { with water (F-W) }\end{array}$ & $10 \times$ & 5.4 & 1.01 & 5.3 \\
& high-shear granulation & $100 \times$ & 8.0 & 1.53 & 12.2 \\
& with water (HS-W) & $10 \times$ & 4.5 & 0.18 & 0.8 \\
\multirow{2}{*}{ SPL } & melt extrusion & $10 \times$ & 6.5 & 0.14 & 0.9 \\
& electrospinning & $10 \times$ & 1.3 & 0.10 & 0.1 \\
\hline
\end{tabular}

\section{Conclusions}

Macropixel analysis can be performed in any cases when 2 dimensional distribution, such as a hyperspectral image, is available. This study demonstrates and underlines the need for novel ways to interpret the homogeneities between different distributions caused by manufacturing technologies in a critical and objective way. Two kind of macropixel analysis methodology has been tested on the real samples, which were prepared by five types of manufacturing technologies including batch and continuous processing. Percentage of homogeneities by means of Poole-index (calculated from non-overlapping macropixels) proved to be a more sensitive measure than conventional DHI (created by CLMB approach) method, when detection of large inhomogeneity differences was required. However, it was not sensitive enough for discriminating the samples produced by continuous methods with fine inhomogeneities. Even more crucially, the DLT method is a complicated and lengthy process due to the determination of the limited macropixel sizes. The CLMB approach is simpler and it requires lower calculation time and less computing power. Consequently, it was desirable to make DHI capable to describe the sample homogeneity more sensitively. Expecting that the DHI can be upgraded (to distinguish the homogeneities of similar samples properly) the use of weighted means and relative standard deviations of original datasets was included in the method. More precise measure of homogeneities could be achieved this way. These modifications enable the DHI method to compare not only similar batches but also maps of totally different distributions exactly in course of content uniformity examinations. In the future, the calculation of Poole-indices becomes unnecessary as the modified DHIs (cDHI) provide perfect description of the sample homogeneity.

\section{Acknowledgement}

This work was financially supported by the Hungarian projects: OTKA PD 108975, OTKA K112644.

\section{References}

[1] Smith, G. P. S., McGoverin, C. M., Fraser, S. J., Gordon, K. C. "Raman imaging of drug delivery systems." Advanced Drug Delivery Reviews. 89, pp. 21-41. 2015. https://doi.org/10.1016/j.addr.2015.01.005

[2] Vajna, B., Pataki, H., Nagy, Z., Farkas, I., Marosi, G. "Characterization of melt extruded and conventional Isoptin formulations using Raman chemical imaging and chemometrics." International Journal of Pharmaceutics. 419(1-2), pp. 107-113. 2011.

https://doi.org/10.1016/j.ijpharm.2011.07.023

[3] Farkas, A., Vajna, B., Sóti, P. L., Nagy, Z. K., Pataki, P., Van der Gucht, F., Marosi, G. "Comparison of multivariate linear regression methods in micro-Raman spectrometric quantitative characterization." Journal of Raman Spectroscopy. 46(6), pp. 566-576. 2015.

https://doi.org/10.1002/jrs.4672

[4] Vajna, B., Bodzay, B., Toldy, A., Farkas, I., Igricz, T., Marosi, G. "Analysis of car shredder polymer waste with Raman mapping and chemometrics." eXPRESS Polymer Letters. 6(2), pp. 107-119. 2012. https://doi.org/10.3144/expresspolymlett.2012.12 
[5] Gendrin C., Roggo Y., Collet C. "Content uniformity of pharmaceutical solid dosage forms by near infrared hyperspectral imaging: A feasibility study." Talanta. 73(4), pp. 733-741. 2007.

https://doi.org/10.1016/j.talanta.2007.04.054

[6] Gergely, S., Izsó, E., Salgó, A. "Comparison of Different Types of Near Infrared (NIR) Instruments in Ability to Measure Alkaloids in Capsule of Poppy (P. somniferum)." Periodica Polytechnica Chemical Engineering. 59(4), pp. 277-282. 2015. https://doi.org/10.3311/PPch.7725

[7] Li, Y., Chow, P. S., Tan, R. B. H. "Quantification of polymorphic impurity in an enantiotropic polymorph system using differential scanning calorimetry, X-ray powder diffraction and Raman spectroscopy." International Journal of Pharmaceutics. 415(1-2), pp. 110-118. 2011. https://doi.org/10.1016/j.ijpharm.2011.05.058

[8] Sacré, P.-Y., Deconinck, E., Saerens, L., De Beer, L., Courselle, P., Vancauwenberghe, R., Chiap, P., Crommen, J., De Beer, J. O. "Detection of counterfeit Viagra ${ }^{\circledR}$ by Raman microspectroscopy imaging and multivariate analysis." Journal of Pharmaceutical and Biomedical Analysis. 56(2), pp. 454-461. 2011. https://doi.org/10.1016/j.jpba.2011.05.042

[9] Sabin, G. P., Lozano, V. A., Rocha, W. F. C., Romão, W., Ortiz, R. S., Poppi, R. J. "Characterization of sildenafil citrate tablets of different sources by near infrared chemical imaging and chemometric tools." Journal of Pharmaceutical and Biomedical Analysis. 85, pp. 207-212. 2013. https://doi.org/10.1016/j.jpba.2013.07.036

[10] Sacré, P.-Y., De Bleye, C., Chavez, P.-F., Netchacovitch, L., Hubert, Ph., Ziemons, E. "Data processing of vibrational chemical imaging for pharmaceutical applications." Journal of Pharmaceutical and Biomedical Analysis. 101, pp. 123-140. 2014.

https://doi.org/10.1016/j.jpba.2014.04.012

[11] Amigo, J. M. "Practical issues of hyperspectral imaging analysis of solid dosage forms." Analytical and Bioanalytical Chemistry. 398(1), pp. 93109. 2010. https://doi.org/10.1007/s00216-010-3828-z

[12] Fan, L. T., Chen, S. J., Watson, C. A. "Solid Mixing." Industrial \& Engineering Chemistry. 62(7), pp. 53-69. 1970.

https://doi.org/10.1021/ie50727a009
[13] Hamad, M. L., Ellison, C. D., Khan, M. A, Lyon, R. C. "Drug product characterization by macropixel analysis of chemical images." Journal of Pharmaceutical Sciences. 96(12), pp. 3390-3401. 2007.

https://doi.org/10.1002/jps.20971

[14] Rosas, J. G., Blanco, M. "A criterion for assessing homogeneity distribution in hyperspectral images. Part 1: Homogeneity index bases and blending processes." Journal of Pharmaceutical and Biomedical Analysis. 70, pp. 680-690. 2012. https://doi.org/10.1016/j.jpba.2012.06.036

[15] Sacré, P.-Y., Lebrun, P., Chavez, P.F., De Bleye, C., Netchacovitch, L., Rozet, E., Klinkenberg, R., Streel, B., Hubert, Ph., Ziemons, E. "A new criterion to assess distributional homogeneity in hyperspectral images of solid pharmaceutical dosage forms." Analytica Chimica Acta. 818, pp. 7-14. 2014. https://doi.org/10.1016/j.aca.2014.02.014

[16] Bobiak, J.-P., McGeorge, G. "Assessing Hyperspectral Image Content of Pharmaceutical Products Using the Herfindahl-Hirschman Index and Ripley’s K-Functions." Applied Spectroscopy. 69, pp. 955-965. 2015. https://doi.org/10.1366/14-07650

[17] Vajna, B., Farkas, I., Szabó, A., Zsigmond, Z., Marosi, G. "Raman microscopic evaluation of technology dependent structural differences in tablets containing imipramine model drug." Journal of Pharmaceutical and Biomedical Analysis. 51(1), pp. 30-38. 2012. https://doi.org/10.1016/j.jpba.2009.07.030

[18] Nagy, Z. K., Balogh, A., Vajna, B., Farkas, A., Patyi, G., Kramarics, Á., Marosi G. "Comparison of electrospun and extruded soluplus ${ }^{\circledR}$-based solid dosage forms of improved dissolution." Journal of Pharmaceutical Sciences. 101(1), pp. 322-332. 2012. https://doi.org/10.1002/jps.22731

[19] Mark, H. "Inverse Least Squares and Classical Least Squares methods for Quantitative Vibrational Spectroscopy." In: Handbook of Vibrational Spectroscopy. Vol. 1 (Chalmers, J. M., Griffiths, P. R. (ed.)), pp 22762292. John Wiley \& Sons, London. 2002.

[20] Rosas, J. G., Blanco, M. "A criterion for assessing homogeneity distribution in hyperspectral images. Part 2: Application of homogeneity indices to solid pharmaceutical dosage forms." Journal of Pharmaceutical and Biomedical Analysis. 70, pp. 691-699. 2012. https://doi.org/10.1016/j.jpba.2012.06.037 